\title{
VISIÓN ARTIFICIAL EN RECONOCIMIENTO DE PATRONES PARA CLASIFICACIÓN DE FRUTAS EN AGRONEGOCIOS
}

\author{
ARTIFICIAL VISION IN PAT'TERN RECOGNITION FOR FRUIT \\ CLASSIFICATION IN AGROBUSINESS \\ (D) Reynaldo Sucari León ${ }^{*}$, (D) Yolanda Aroquipa Durán ${ }^{1}$, (D) Edgardo Quispe Yapo², (D) Anibal \\ Sucari León ${ }^{2}$, Luz Delia Quina Quina ${ }^{3}$, (D) Fredy Abel Huanca Torres ${ }^{4}$ \\ rsucari@unah.edu.pe; yaroquipa@unah.edu.pe; gardo25@hotmail.com; anibalsl@hotmail.com; \\ dquina899@unajma.edu.pe; abel.huanca@upeu.edu.pe \\ 1Universidad Nacional Autónoma de Huanta, Ayacucho, Perú \\ 2Universidad Nacional del Altiplano, Puno, Perú. \\ ${ }^{3}$ Universidad Nacional José María Arguedas, Apurímac, Perú \\ ${ }^{4}$ Universidad Peruana Unión, Lima, Perú \\ *Correspondencia: Reynaldo Sucari León. Email: rsucari@unah.edu.pe
}

Recibido: 21.03.2020 | Aprobado: 09.04.2020

\section{RESUMEN}

La presente tuvo como objetivo determinar la efectividad de aplicar visión artificial en reconocimiento de patrones para la clasificación de frutas en los agronegocios, para ello se ha empleado una base de datos con 50 registros de 6 variedades de frutas donde se consideró 4 características para cada fruta y una muestra de 20 frutas, así mismo se ha empleado la técnica reconocimiento automático de patrones por medio del clasificador bayesiano implementado en Octave, en el experimento se logró reconocer las frutas hasta en un $93.33 \%$ y errando en $6.67 \%$. Concluyendo que si es efectivo aplicar la visión artificial en el reconocimiento de patrones para clasificar frutas.

Palabras clave: Clasificación de frutas, reconocimiento de patrones y visión artificial.

\section{ABSTRACT}

The purpose of this research was to determine the effectivity of applying artificial vision on patterns recognition for the fruits classification in agrobusiness, for this purpose we has used a database with 50 records of 6 fruit varieties with 4 characteristics that are considered for each fruit and a sample of 20 fruits, likewise has been used the automatic pattern recognition technique through the Bayesian classifier implemented in Octave, in the experiment it was recognized to the fruits up to $93.33 \%$ and erring in other cases $6.67 \%$. Concluding that is effective to apply artificial vision in the pattern recognition classify fruits.

Keywords: Fruit classification, pattern recognition and artificial vision. 


\section{INTRODUCCIÓN}

Todo sistema de visión por computador, al cual se conoce como visión artificial, en la actualidad se emplean cada vez más en procesos de reconocimiento automático ya sea en industria agroalimentaria o agroexportadora, estas técnicas forman parte de las herramientas indispensables para dichas empresas para mantenerse en un nivel competitivo (Granados Montelongo, 2000). Estas técnicas se emplean en la clasificación y supervisión de calidad de productos, ya que estos sistemas ofrecen el potencial necesario para automatizar las prácticas manuales de selección, a la vez estandarizan las técnicas reduciendo las costosas tareas humanas de clasificación e inspección, a pesar de conocer las limitaciones humanas y la susceptibilidad a cometer errores. Así mismo, el desarrollo de tecnologías digitales aplicadas en la agricultura otorga nuevas posibilidades para automatizar algunos procesos tecnológicos (Rodriguez Pérez, 2015).

Respecto a la visión artificial, Domingo (2004) sostiene que el término visión por computadora, engloba muchos constructos, como procesamiento digital de imágenes; proceso que permite tomar una imagen y reproducirla bajo ciertas características, análisis de imágenes; también Elamvazuthi, Sinnadurai, Khan, y Vasant (2009) consideran procesos como el ordenamiento, etiquetado y empaquetado; dichos procesos permiten obtener mediciones, interpretaciones o decisiones, reconocimiento de patrones; proceso por el cual se asigna un objeto a una clase a partir la medición de otros objetos. También

Cubero García (2012) indica que los sistemas que emplean la tecnología, en la actualidad permiten capturar imágenes de manera simultánea empleando cámaras para luego analizarla, optimizando considerablemente el tiempo en los procesos de selección de las frutas.

También por su parte, Porras, De la Cruz, y Morán (2014) investigaron un sistema automatizado controlado por visión artificial y a su vez empleaba sensor de luz, motor paso a paso y Matlab, indicando que el principio básico para su funcionamiento fue el transporte de objetos de color mediante una faja transportadora, para luego ser detectados por un sensor, capturados por una cámara web, y procesador en Matlab que los clasificó en distintos contenedores logrando una clasificación eficaz ya sea en color así como de forma. Para Cáceres (2014) la visión artificial es una parte importante de la inteligencia artificial, y de hecho su aplicación al tratamiento de imágenes en formato binario, concluyendo que es posible la adquisición automática de imágenes sin tener contacto físico, entre las aplicaciones destacadas fueron en control de calidad, manipulación de materiales, calibración de aparatos, monitorización de procesos, selección y clasificación de objetos. Otro estudio indica que la estrategia de visión por computadora es usada para reconocer una fruta mediante 4 características básicas, como: intensidad, color, forma y textura, para ello se ha empleado la técnica del clasificador de mínima distancia, para el experimento se ha usado una base de datos con 2635 frutas de 15 diferentes clases confirmado su eficiencia en la clasificación. (Arivazhagan, Newlin, Selva, y Ganesan, 2010)

Por otra parte, el reconocimiento de patrones es considerado como la disciplina científica que se emplea en la clasificación de objetos que cuentan con un número de categorías o clases, además de ello forma parte integral de muchos sistemas inteligentes 
(Theodoridis y Koutroumbas, 2003). Así mismo, investigadores como Montoya Holguín, Cortés Osorio, y Chaves Osorio (2014) construyeron un prototipo electrónico que logró identificar y clasificar frutas en los supermercados, para ello emplearon las técnicas bayesianas y la técnica del vecino más cercano, logrando con ello obtener resultados con una exactitud igual al 90\% en las pruebas realizadas. A pesar de que en una fruta se pueden realizar múltiples mediciones, no es posible contar con todos los parámetros, por ello solo se alcanza una solución importante pero no completa (Pencue-Fierro y León-Téllez, 2003)

En esta misma línea se cuenta con las investigaciones que abordaron los algoritmos de clasificación del tipo supervisado (Montoya Holguín et al., 2014), el autor también utilizó dos modelos de clasificación, como el algoritmo KNN, basado en la ubicación de los vecinos más cercanos y el algoritmo de clasificación probabilístico, basado en el teorema de Bayes. Arivazhagan et al. (2010) aplicaron indicadores de estadística descriptiva como mecanismo de reconocimiento de frutas, obteniendo imágenes con espacio de color HSV y estadísticos, como la media, desviación estándar, sesgo y curtosis los que son derivados de los componentes $\mathrm{H}$ y S. El algoritmo para extraer la región de interés. Finalmente, Gatica, Best, Ceroni y Lefranc (2013) describen un proceso de detección de bordes y selección de candidatos, en el que puede haber un error latente, especialmente si el proceso está automatizado. Es necesario tener dos etapas de decisión, uno centrado en discernir si el candidato es una aceituna y para determinar el número de aceitunas, teniendo en cuenta la superposición de ellos, en función de las propiedades geométricas y morfológicas de los especímenes que se solapan. Para cumplir estos requisitos se utiliza una Red Neuronal Artificial Feedforward, con un algoritmo de aprendizaje de retro propagación.

Sandoval y Prieto (2009) también desarrollaron un sistema que emplea técnicas de visión artificial, pero esta vez, para la clasificación de frutos de café considerando ocho categorías, ello según su madurez. Tomaron medidas de forma, color y textura que fueron obtenidas al analizar una imagen del fruto, logrando implementar un clasificador Bayesiano válido al $96.88 \%$ contrastado por el método de validación cruzada. Por su parte, Aldabas Rubira (2002) empleó una red neuronal artificial multinivel para el reconocimiento óptico de caracteres OCR; que viene a formar parte del reconocimiento de patrones, el código implementado se realizó con Matlab entrenado por el algoritmo back propagation. En esta misma línea; Vega, Cortez, Huayna, Alarcón y Romero (2009) indican que, en la actualidad, la inteligencia artificial brinda soluciones en varios aspectos de las actividades humanas, siendo estas en la economía, medicina, física, administración y entre otras. Una de las técnicas que alcanzó una importancia trascendental son las redes neuronales, en aplicaciones de reconocimiento de patrones, comprobando su alto nivel de efectividad en el reconocimiento de números.

En este sentido, cabe resaltar los aportes de los investigadores de la UNMSM como Vega y Cortez (2010) quienes desarrollaron una red neuronal para el reconocimiento de frutas basado en una red que ya era capaz de reconocer números, para ello trabajaron con plátanos y naranjas, además de ello cada uno con dos modelos diferentes. Así mismo, Pencue-Fierro y León-Téllez (2003) han demostrado la factibilidad de desarrollar sistemas de visión artificial con aplicaciones respecto a las exigencias regionales alcanzando niveles de efectividad superiores al 90\%. También Gatica, Best, Ceroni y Lefranc (2013) 
presentaron un nuevo método para el reconocimiento de frutos de olivo, para ello emplearon el tamaño, peso y color como características que fueron implementadas en una red neuronal como parte de la solución, logrando reconocer los frutos en dos estados; peso y tamaño para estimar el mejor momento de la cosecha y el color como característica para el reconocimiento del fruto.

Por otra parte, un Clasificador Bayesiano para autores como Duda, Hart, y Stork (2001) es una técnica estadística que se basa en la teoría de Bayes del error mínimo, así cualquier objeto con características previamente determinadas, se afirma que pertenece a una clase si y solo si la probabilidad de pertenecer a esta clase es mayor que la probabilidad de pertenecer a otra clase. Un clasificador permite clasificar ciertos patrones para tomar una decisión frente a una situación dada. Dicho clasificador bayesiano, está basado en una función de densidad normal multivariada, ya que se tratan muchas variables y varias clases a la vez.

La función de densidad normal multivariada en d dimensiones está dada por:

$$
P(\mathrm{x})=\frac{1}{(2 \pi)^{d / 2}\left|\sum\right|^{1 / 2}} \exp \left[-\frac{1}{2}(\mathrm{x}-\mu)^{t} \sum^{-1}(\mathrm{x}-\mu)\right]
$$

Donde:

$x=\left(x_{1}, x_{2}, \ldots, x_{d}\right)^{t}(\mathrm{t}$ se define en forma de vector transpuesto $)$

$\mu=\left(\mu_{1}, \mu_{2}, \ldots, \mu_{d}\right)^{t}$ vector media

$\Sigma=d^{*} d$ matriz de covariancia

$|\Sigma|$ y $\Sigma^{-1}$ son la determinante y la inversa, respectivamente

Respecto al agronegocio, el desarrollo humano es consecuencia de la calidad total, así como de la sostenibilidad en los agronegocios, por ello, las organizaciones para lograr una mejora continua en la productividad y la autorrealización de su personal deben promover el aprendizaje y su aplicación del conocimiento mediante las tecnologías existentes. (Fernández, 2000)

En este sentido, el objetivo general de la investigación fue determinar la efectividad de aplicar visión artificial en el reconocimiento de patrones para la clasificación de frutas en los agronegocios.

\section{MATERIALES Y MÉTODOS}

Tipo, diseño y nivel

El presente estudio corresponde al tipo de investigación aplicada; dado que está centrado en la aplicación de la teoría para la solución del problema de clasificación de frutas, todo ello enmarcado en el enfoque cuantitativo, que siguió un diseño experimental de corte longitudinal y que se halla dentro de un nivel explicativo.

\section{Población muestra}

Para realizar la prueba del clasificador de frutas mediante visión artificial, se consideró las frutas que se expenden en el mercado provincial de Huanta - Ayacucho, considerando así una población muy grande de frutas. 


\section{Tabla 1}

Muestra de frutas para la investigación

\begin{tabular}{clcc}
\hline $\mathbf{N}^{\mathbf{o}}$ & \multicolumn{1}{c}{ Fruta } & Muestra & Porcentaje \\
\hline 1 & Palta & 20 & 16.67 \\
2 & Tuna & 20 & 16.67 \\
3 & Pera & 20 & 16.67 \\
4 & Mango & 20 & 16.67 \\
5 & Naranja & 20 & 16.67 \\
6 & Granadilla & 20 & 16.67 \\
\hline & TOTAL & $\mathbf{1 2 0}$ & $\mathbf{1 0 0 . 0 0}$ \\
\hline
\end{tabular}

\section{Técnicas e Instrumentos}

En la recolección de los datos, la técnica que fue aplicada es la observación y se ha empleado una ficha de datos como instrumento; la ficha de datos consideró el nombre de la fruta con sus respectivas características físicas como: tamaño, forma, color y textura, que luego generó una base de conocimientos.

\section{Procedimientos estadísticos}

El análisis de la información recabada respecto a las características de las frutas, fueron ingresadas y alimentadas en una base de datos mediante un archivo de texto, que posteriormente fue invocado por el código del programa realizado en Octave; realizando un análisis multivariado y probabilístico cumpliendo las reglas del teorema de Bayes, todo ello posteriormente al procesamiento de imagen de la fruta que se desea seleccionar. Así mismo se emplearon cuadros de distribución de frecuencias y análisis proporcional de datos.

\section{RESULTADOS Y DISCUSION}

En la presente investigación, guiados por el objetivo general la cual fue determinar la efectividad de aplicar visión artificial en el reconocimiento de patrones para la clasificación de frutas en los agronegocios, se han obtenido los siguientes resultados.

Referente al tratamiento de las imágenes de las frutas consideradas en la investigación, se utilizó la técnica de segmentación; mediante umbral sobre la descomposición de capas de la imagen captada, en esta oportunidad la capa de color azul del modelo RGB (sigla en inglés de red, green, blue, en español "rojo, verde y azul") aprovechando así la circunstancia de tener un fondo blanco.

\section{Figura 1.}

Capa azul de la imagen de una palta

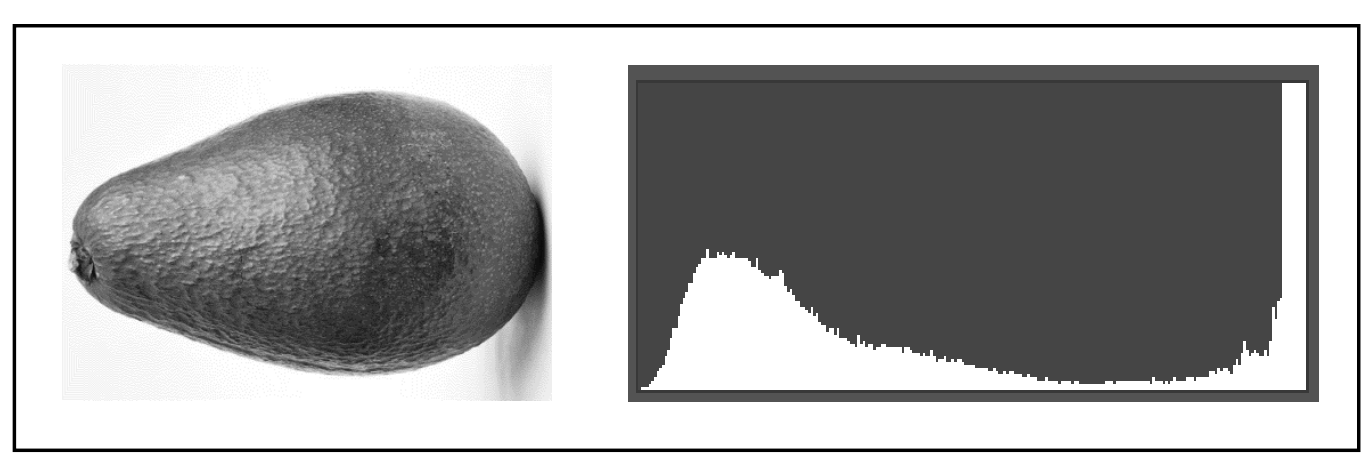


Seguidamente se realiza el análisis en el modelo RGB de la imagen; el cual consiste en obtener el valor umbral y proceder con la binarización global de la capa azul; ello se consigue con la función im2bw de Octave implementada inicialmente en MatLab, ésta transforma la imagen de capa azul del modelo RGB en una nueva imagen binaria ( 1 y 0 ) con base en el umbral calculado anteriormente. Luego mediante el proceso de recuperación de la imagen en función de la multiplicación de la imagen binarizada por las imágenes de cada canal de color RGB, esto se logra invirtiendo los valores de los bits de la imagen binaria inicial y así se obtiene la nueva máscara, como se ilustra en la siguiente figura.

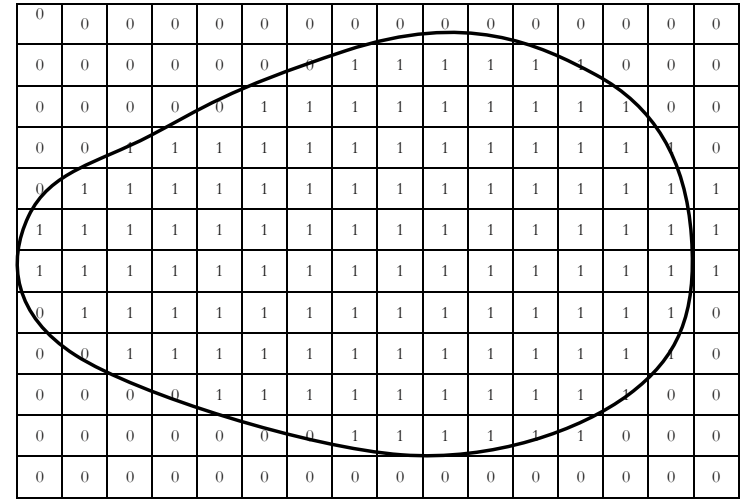

Figura 2. Imagen binaria con bits.

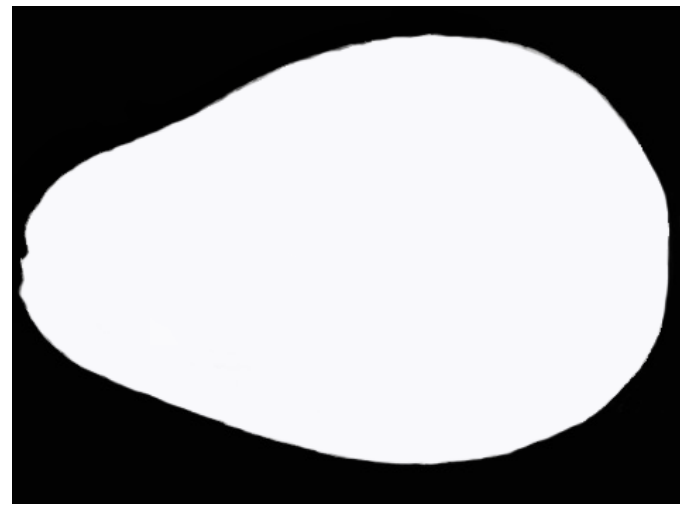

Figura 3. Imagen binaria con bits invertidos.

Seguidamente con la máscara, mostrada en la Figura 3, es posible eliminar los pixeles del fondo y obtener los pixeles que componen la fruta, realizando básicamente una multiplicación entre ella y cada una de las capas y luego concatenándolas nuevamente para formar la imagen a color, con lo que se obtiene la imagen de la fruta segmentada como se observa en la Figura 4.

\section{Figura 4}

Imagen segmentada de una palta en el modelo RGB.

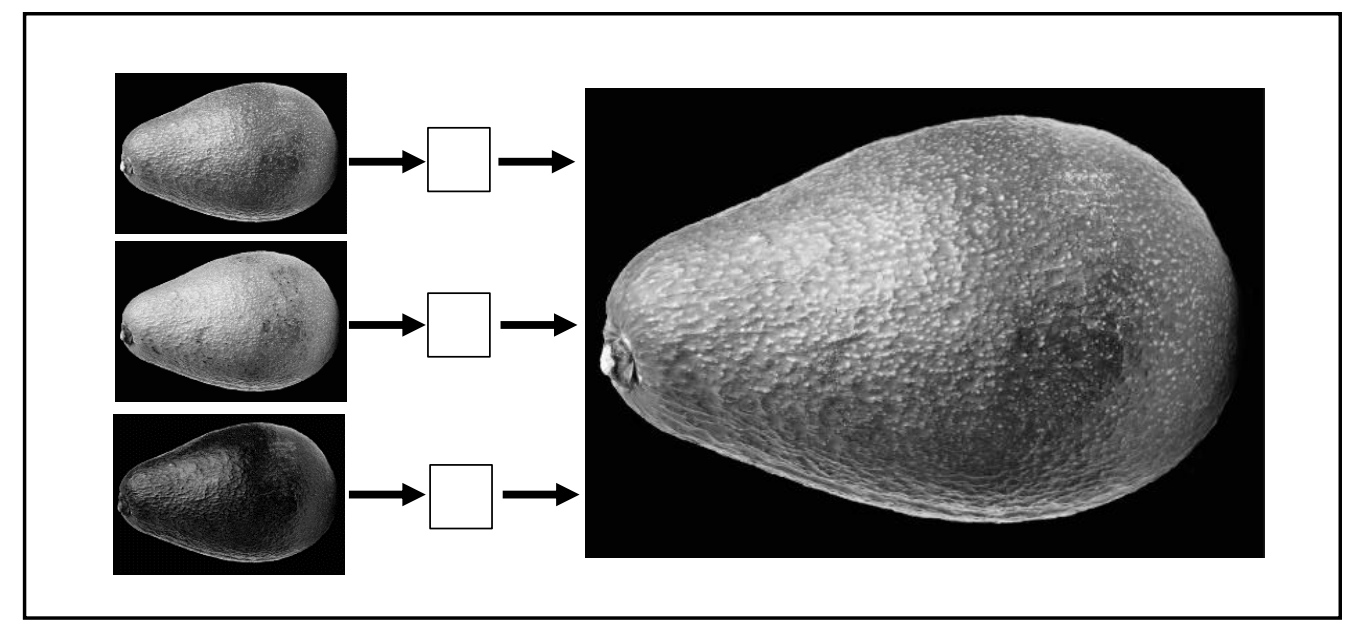

El proceso de descripción, el que consiste en obtener los patrones determinantes que permitieran diferenciar un tipo de fruta (clase) de otro distinto, se tuvieron en cuenta el tamaño, forma, color, textura. Las distintas características utilizadas para medir estas variables fueron: el área, el perímetro, la redondez, el eje mayor, el eje menor y el valor 
medio de los canales HSV y RGB, este procedimiento permitió obtener imágenes como insumo para los algoritmos de clasificación, en nuestro caso el clasificador bayesiano.

\section{Figura 5}

Imágenes de frutas en el modelo RGB.

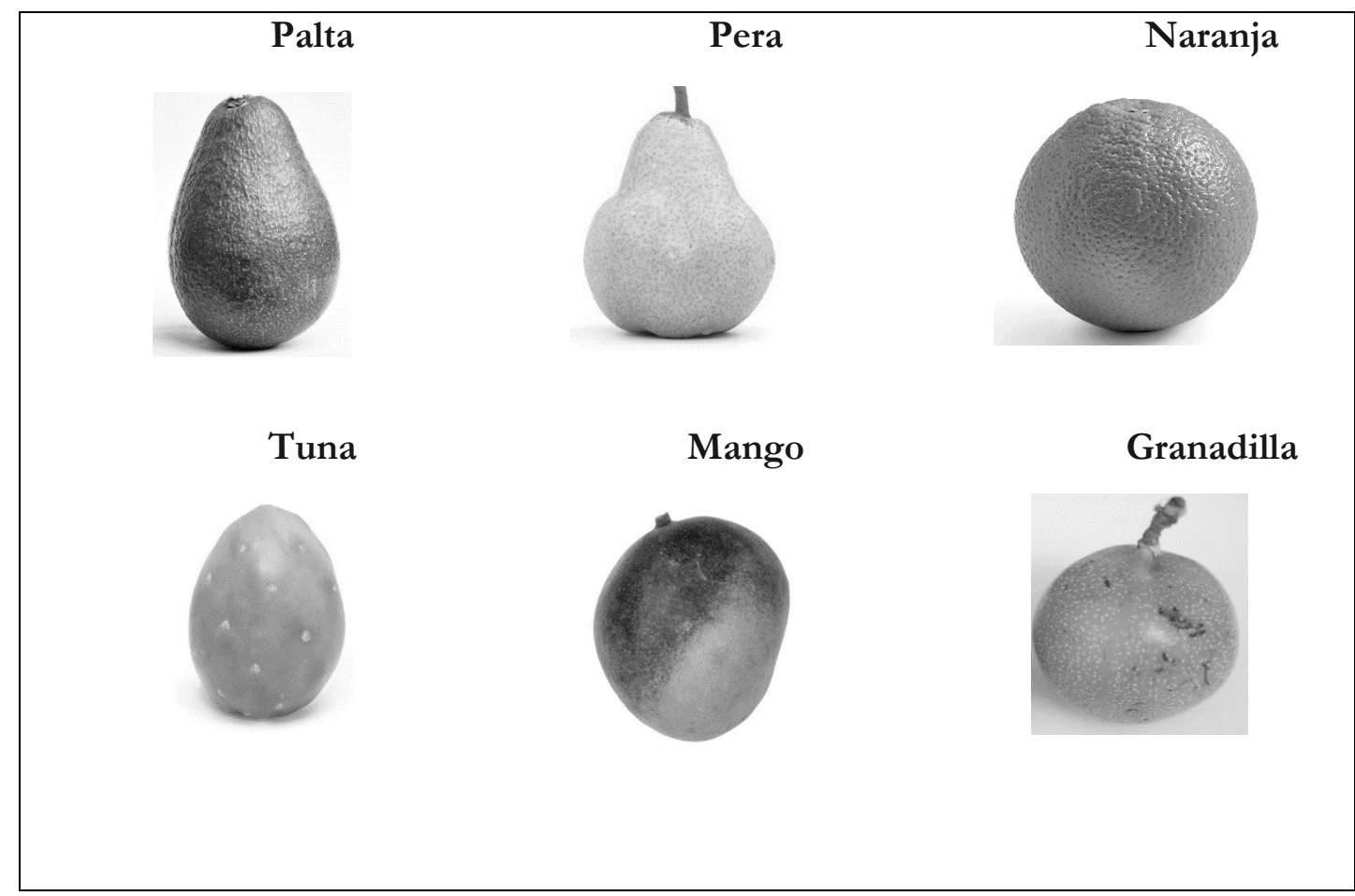

El procedimiento realizado en la presente investigación concuerda con lo planteado por Arivazhagan et al. (2010) cuando proponen para el reconocimiento artificial de una fruta, cuatro variables básicas que caracterizan al objeto, así como la intensidad, color, forma y textura, enfatizando en el color y textura. Estas exigencias se deben superar para que el sistema pueda realizar el reconocimiento automático del tipo de fruta con las imágenes obtenidas por la cámara, muchos tipos de frutas están sujetos a variaciones significativas en color y textura, dependiendo su estado de madurez, por ejemplo, los mangos, y granadillas de color verde uniforme a anaranjado, y las tunas de color verde a otros colores, generando un espectro de colores. Las características de textura se calculan a partir del canal de luminancia ' $\mathrm{V}$ ', y las características de color se calculan a partir de los canales de crominancia ' $\mathrm{H}$ ' y ' $\mathrm{S}$ '. El componente que corresponde al brillo del color (V) se descompuso usando la transformada Wavelet Discreta y la matriz de co-ocurrencia se construye a partir de la sub-banda.

Por otro lado, en la investigación realizada por Gatica et al. (2013) centra principalmente para el tratamiento de imágenes, la extracción del fondo, por medio del análisis del histograma de la imagen, ello utiliza para verificar la posibilidad de separación de los estratos y comprobar los datos establecidos en el análisis de normalidad. Los estratos elegidos para el análisis son: aceituna, tipo de hoja de color verde oscuro, verde claro, y tallo. Realizar un análisis estadístico verifica la normalidad de los datos en cada estrato, considerado como variables aleatorias en virtud de una distribución normal de los datos. 
Seguidamente la imagen hallada es comparada con cada una de las imágenes que están en la base de conocimientos, almacenados previamente para nuestro clasificador bayesiano, para ello previamente se tiene como información de entrada los atributos de la fruta a seleccionar dentro de una de las clases que se tiene dentro de los patrones. El resultado final se muestra en la siguiente tabla:

\section{Tabla 2}

Porcentaje de reconocimiento de frutas por el clasificador bayesiano

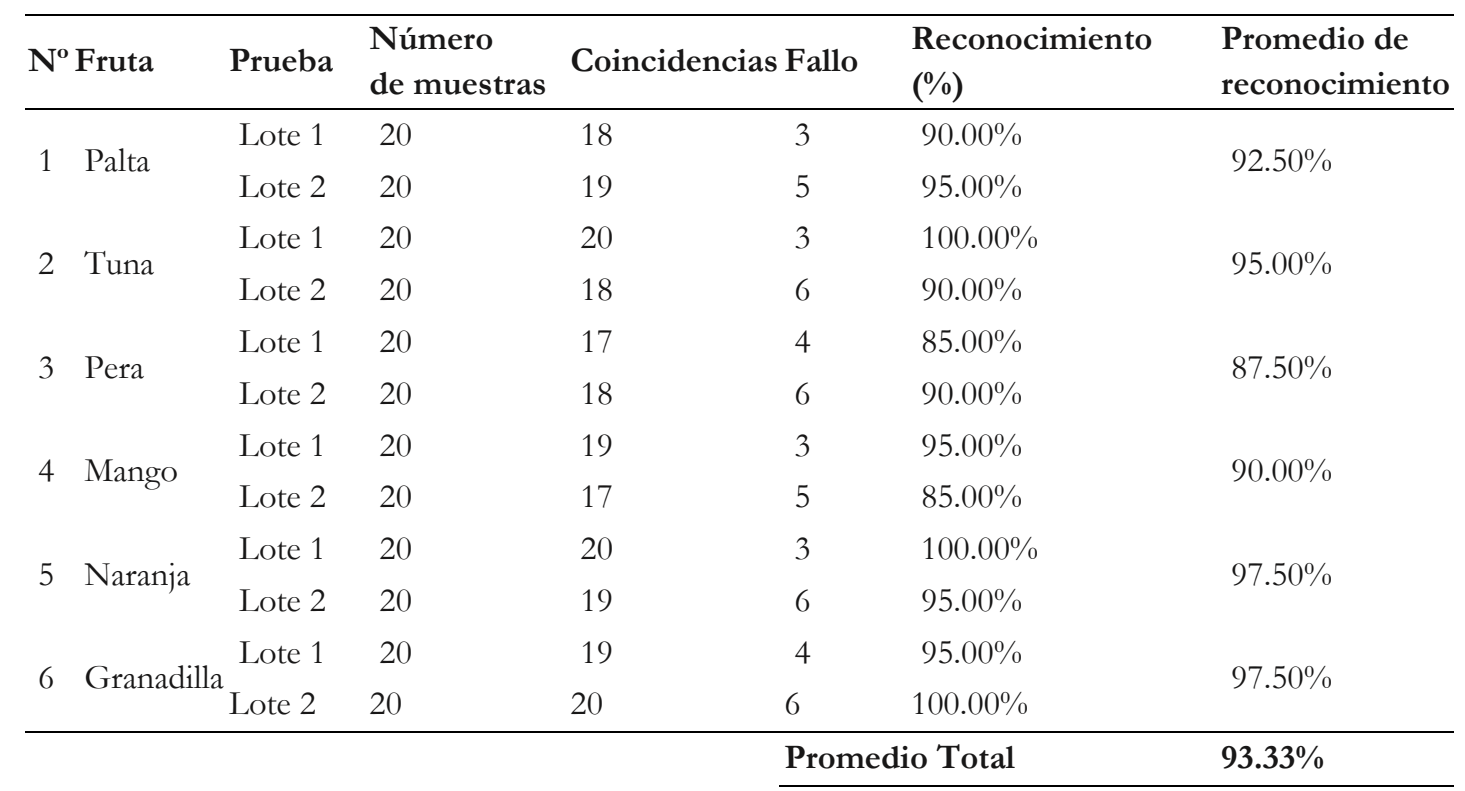

En la tabla 2, se muestra el promedio de reconocimiento para cada una de las frutas, ello se realizó con dos sub muestras, cada una con 20 repeticiones, observando que la máxima efectividad se alcanza con las frutas naranja y granadilla $(97.5 \%)$, seguido de la tuna $(95 \%)$, la palta $(92.5 \%)$ y el más bajo de todos se produce con la pera $(87.5 \%)$, y en promedio global el clasificador bayesiano con visión artificial alcanza un $93.33 \%$ de efectividad.

Cabe resaltar en esta sección, que el procesamiento de imágenes es uno de los aspectos que requiere un tratamiento especial, así como el uso de los modelos de colores donde juega un rol fundamental, al respecto Montoya Holguín et al. (2014) han obtenido mayor éxito al realizar la segmentación de la imagen en canales del modelo RGB y HSV, para la separación del objeto del fondo de la imagen. Particularmente Gatica et al. (2013) centraron sus esfuerzos en la extracción del fondo de la imagen, proceso también basado en la separación por canales del modelo RGB para el análisis del comportamiento espectral de todas las capas establecidas por el contexto. Pero muy diferente a ellos Arivazhagan et al. (2010) empleó el procesamiento de imágenes haciendo uso del modelo HSV para obtener características de textura que fue calculada a partir de ' $V$ ' el canal de luminancia, y las características de color se calcularon a partir de los canales de crominancia ' $\mathrm{H}$ ' y ' $\mathrm{S}$ ', lo que evidencia que este método de procesamiento de segmentación de imágenes ha permitido resultados bastante alentadores.

Referente al proceso del reconocimiento de frutas, el clasificador bayesiano han logrado resultados de éxito superiores al 90\% en el reconocimiento de frutas (Montoya 
Holguín et al., 2014) y (Elamvazuthi et al., 2009) particularmente el primero de ellos, al realizar una comparación entre el rendimiento del algoritmo KNN y Bayesiano, este último algoritmo mostró mejor efectividad que $\mathrm{KNN}$, otros resultados obtenidos fueron muy buenos al usar el algoritmo bayesiano (Pencue-Fierro \& León-Téllez, 2003) y estos son distintos, pero no mejores al utilizar comparaciones de indicadores estadísticos como la media, desviación estándar, sesgo y curtosis aplicados a los canales $\mathrm{H}$ y S del modelo de color HSV (Arivazhagan et al., 2010). Este autor realizó estudios basados principalmente en el color y textura, logrando obtener hasta un $95 \%$ de precisión en el reconocimiento de frutas. Por otra parte, se cuenta con resultados obtenidos por la primera red neuronal fue buena con el $97 \%$ clasificación correcta y la segunda red alcanzó un rendimiento de 88,8\% (Gatica et al., 2013).

\section{CONCLUSIONES}

La visión artificial en la actualidad constituye una de las técnicas más utilizada para la identificación y clasificación de frutas, que podría ser extendido hacia los vegetales, además está teniendo mayor auge en los últimos años por su versatilidad, aplicabilidad, rapidez y limpieza, es así que se están demostrando su validez en muchas empresas de agronegocios.

El empleo de métodos de procesamiento de imágenes y algoritmos de clasificación como el clasificador bayesiano, es relativo a la aplicación específica; por lo tanto, no se tiene suficiente evidencia para dar juicio y afirmar que algún proceso de reconocimiento automático es mejor que otro, dado que la precisión en el reconocimiento de productos como frutas y vegetales están por encima del 85\%; ya que, en algunos contextos es un éxito completo.

\section{REFERENCIAS BIBLIOGRÁFICAS}

Aldabas Rubira, E. (2002). Introducción al reconocimiento de patrones mediante redes neuronales. IX Jornades de Conferencies d'Enginyeria. Retrieved from http://petrus.upc.es/ microele/neuronal/xn/

Arivazhagan, S., Newlin Shebiah, R., Selva Nidhyanandhan, S., \& Ganesan, L. (2010). Fruit Recognition using Color and Texture Features. In Journal of Emerging Trends in Computing and Information Sciences (Vol. 1). Retrieved from http:/ / www.cisjournal.org

Cáceres Tello, J. (2014). La visión artificial y las operaciones morfológicas en imágenes binarias. Retrieved from http://www.um.es/waspssViewproject

Cubero García, S. (2012). Diseño e implementación de nuevas tecnologías basadas en visión artificial para la inspección no destructiva de la calidad de fruta en campo y minimamente procesada. Universidad Politécnica de Valencia.

Domingo, M. (2004). Visión por Computador. Retrieved from http://www.ing.puc.cl/

Duda, R. O., Hart, P. E., \& Stork, D. G. (2001). Pattern Classification - Stork - Google Libros. $\quad$ Retrieved November 10, 2019, from https://books.google.com.pe/books?hl=es\&lr=\&id=Br33IRC3PkQC\&oi=fnd\& $\mathrm{pg}=$ PR3\&ots $=2 \mathrm{xDRMtd} 6 \mathrm{Hp} \& \operatorname{sig}=\mathrm{euCrNoI04hncVOlqnKDDbZnKTMs \& redir}$ _esc $=\mathrm{y} \# \mathrm{v}=$ onepage $\& \mathrm{q} \& \mathrm{f}=$ false 
Elamvazuthi, I., Sinnadurai, R., Khan, A., \& Vasant, P. (2009). Fruit Sorting using Fuzzy Logic Techniques. AIP Conference Proceedings, 225-230.

Fernández Brondo, J. M. (2000). El desarrollo humano como base en la calidad total y la sostenibilidad en los agronegocios. Revista Mexicana de Agronegocios, IV(7), 79-92. Retrieved from http://www.redalyc.org/articulo.oa?id=14107411

Gatica, G., Best, S., Ceroni, J., \& Lefranc, G. (2013). Olive fruits recognition using neural networks. Procedia Computer Science, 17, 412-419. https://doi.org/10.1016/j.procs.2013.05.053

Granados Montelongo, J. A. (2000). Fundamentos de Calidad Total en los Agronegocios. Revista Mexicana de Agronegocios, IV, 476-484.

Montoya Holguín, C., Cortés Osorio, J. A., \& Chaves Osorio, J. A. (2014). Automatic recognition system of fruits based computer vision. In Revista chilena de ingeniería (Vol. 22).

Pencue-Fierro, E. L., \& León-Téllez, J. (2003). Detección y clasificación de defectos en frutas mediante el procesamiento digital de imágenes. Revista Comlombiana de Física, 35(1), 148-151.

Porras, J., De la Cruz, M., \& Morán, A. (2014). Clasification system based on computer vision. Retrieved from http://v-beta.urp.edu.pe/pdf/id/2881/n/clasification-systembased-on-computer-vision

Rodriguez Pérez, F. (2015). Sistema automatizado para la determinación del estado de maduración en frutas bomba. Revista Ciencias Técnicas Agropecuarias, 24, 56-61. Retrieved from http://www.redalyc.org/articulo.oa?id=93243475010

Sandoval, Z., \& Prieto, F. (2009). Procesamiento de imágenes para la clasificación de café cereza. Prospectiva, 7(1), 67-73.

Theodoridis, S., \& Koutroumbas, K. (2003). Pattern Recognition (Second; Elsevier, Ed.). USA.

Vega Huerta, H., \& Cortez Vásquez, A. (2010). Entrenamiento de Redes Neuronales para el Reconocimiento de Frutas. Paradigmas 2, 1, 141-164.

Vega Huerta, H., Cortez Vásquez, A., Huayna, A. M., Alarcón Loayza, L., \& Romero Naupari, P. (2009). Reconocimiento de patrones mediante redes neuronales artificiales. Revista de Ingeniería de Sistemas e Informática, 6(2), 17-26.

\section{CITAR COMO:}

Sucari León, R., Aroquipa Durán, Y., Quina Quina, L. D., Quispe Yapo, E., Sucari León, A., \& Huanca Torres, F. A. s. (2020). Visión artificial en reconocimiento de patrones para clasificación de frutas en agronegocios. Puriq, 2(2), 109-118. https://doi.org/10.37073/puriq.2.2.76 